\title{
SCHEDULE OF THE COLLOQUIUM
}

Beijing, 1992

SUNDAY, SEPT. 6

$\begin{array}{ll}\text { 08:30 AM-04:30 PM } & \begin{array}{l}\text { Excursion to the Great Wall and Huairou Solar } \\ \text { Observing Station }\end{array} \\ \text { 06:30-08:30 PM } & \text { Informal Reception }\end{array}$

MONDAY, SEPT. 7

09:00-12:20 AM

Colloquium Session 1: STRUCTURE OF ACTIVE REGIONS

Chairman, H. Zirin

Welcoming Address:

09:00-09:10 Zhou Guangzhao, President, Chinese Academy of Sciences

Speech of Congratulations:

09:10-09:20 Chen Jiaer, Vice President, National Nature Science Foundation of China

Invited Papers:

09:20-09:50 A. Title:

Evolution and Structure of Fine Structure Magnetic Fields

09:50-10:20 A. Hofmann, W. Schmidt and T. D. Tarbell:

High Resolution Magnetic Field Measurements in the Sunspot Photosphere

10:20-10:50 C. U. Keller:

Small-scale Structures in Active Regions

10:50-11:05 Tea Break

Oral Contributed Papers:

11:05-11:20 M. Sobotka, J. A. Bonet, M. Vazquez:

High-resolution Observations of Umbral Fine Structure

11:20-11:35 J. Sanchez Almeida, V. Martinez Pillet, J. Trujillo Bueno, B. W. Lite: High Angular Resolution Stokes V Spectra in Penumbrae

11:35-11:50 S. I. Gopasyuk:

Changes with Height of Longitudinal Magnetic Field in Active Regions

11:50-12:05 N. Mein, P. Mein, B. Schmieder, P. Demoulin, Guoxiang Ai, and O. Engvold:

Active Region Evolution Through Coordinated Observations 


\section{2:00-5:00 PM}

Colloquium Session 2: THEORY OF ACTIVE REGION STRUCTURE Chairman, P. R. Wilson

\section{Invited Papers:}

02:00-02:30 T. Sakurai:

Computational Modeling of Solar Magnetic Fields

02:30-03:00 S. T. Wu:

Extropolation of Non Linear Force Free Field: A Numerical Model

\section{Oral Contributed Papers:}

03:00-03:15 K. Petrovay:

The Small-scale Photospheric Magnetic Field as an Indicator of the Dynamo

03:15-03:45 Tea Break

03:45-04:00 Edward Lu:

Avalanches of Magnetic Reconnection

04:00-04:15 E. I. Mogilevsky:

Energy in the Magnetic Topological Centers of the Active Regions

04:15-04:30 Shi-hui Ye, Jie-hai Jin:

Monochromatic Images of Sunspots in Linearly Polarized Radiation and Structure of Their Magnetic Fields

04:30-04:45 Li Sang Jae:

The Effects of Longitudinal Magnetic Field and Hall Current on the Thermal Instability of the Current Sheet and the Trigger for Solar Flares

04:45-05:00 Suresh Chandra and Lalan Prasad:

Force-free Magnetic Fields May Have a "Loss of Equilibrium"

\section{7:00-09:30 PM Excursion}

Beijing Opera

\section{TUESDAY, SEPT.8}

08:45-12:00 AM

Colloquium Session 3: TECHNIQUES OF MAGNETIC FIELD MEASUREMENTS

Chairman, J. Stenflo

Invited Papers:

08:45-09:15 Guoxiang Ai:

Progress in Measurements of Solar Magnetic Fields

09:15-09:45 H. Jones:

Spectrometer-Based Magnetographs

09:45-10:15 D. Deming:

$12 \mu \mathrm{m}$ Magnetic Field Measurements

10:15-10:30 Tea Break 
Oral Contributed Papers:

10:30-10:45 K. Ichimoto, T. Sakurai, Y. Nishino, K. Shinoda, M. Noguchi. K. Kumagai, H. Imai, M. Irie, M. Miyashita, N. Tanaka, I. Sano and Z. Zhao: Magnetic Field Observations with the Solar Flare Telescope at Mitaka

10:45-11:00 P. Mine, J. Rayrole:

Themis Telescope Prospects in High Resolution Magnetic Field Observations

11:00-11:15 Rob Rutten and Mats Carlsson:

Infrared Rydberg Magnetometry

11:15-11:30 V. Martinez Pillet, J. Trujillo Bueno et al:

Atomic Orientation in Chromospheric Lines

11:30-11:45 J. Sanchez Almeida, B. W. Lites:

Asymmetric Stokes Q, U and V Line Profiles Observed in Sunspots

11:45-12:00 Zhongquan Qu, Youji Ding, Jiayu Xuan and Shi-hui Ye:

On the Inference of Magnetic Field Vectors From Stokes Profiles: A Generalization of the weak-field Approximation

02:00-05:00 PM Excursion

Shopping Downtown, Friendship Store

07:00-09:30 PM

Colloquium Session 4: FRIENDLY DEBATE-RELATION OF QUIET SUN TO ACTIVE REGIONS

Chairman, R. Howard

Invited Papers:

07:00-07:30 J. Stenflo:

Strong and Weak Magnetic Fields: Nature of the Small-scale Flux Elements

07:30-08:00 H. Zirin:

Strong and Weak Magnetic Fields: Nature of the Small-scale Flux Elements

08:00-09:30 Free Discussion

WEDNESDAY, SEPT. 9 Excursion

08:30-11:30 AM

Beijing Ancient Observatory and Temple of Heaven

01:30-04:30 PM

Forbidden City

THURSDAY, SEPT. 10

08:45-12:00 AM

Colloquium Session 5: FIELDS IN THE CHROMOSPHERE AND CORONA

Chairman, M. Kundu

Invited Papers:

08:45-09:15 S. Tsuneta:

Yohkoh Observations of Active Regions 
09:15-09:45 A. Kruger:

Coronal Magnetic Fields Deduced From Radio Methods

09:45-10:15 T. Sakao:

Hard and Soft X-ray Imaging Observations by YOHKOH of the 1.5 November, 1991 Flare

10:15-10:45 Tea Break

Oral Contributed Papers:

10:45-11:00 Hongqi Zhang, Guoxiang $\mathrm{Ai}$, Wei $\mathrm{Li}$ and Jimin Chen:

Structures of Chromospheric Magnetic Field in Solar Active Regions

11:00-11:15 Wei Li, Guoxiang $\mathrm{Ai}$ and Hongqi Zhang:

The Evolution Features of $\mathrm{H}_{\beta}$ Doppler Velocity Field in Flare Productive Regions

11:15-11:30 S. Enome, H. Nakajima, K. Shibasaki, M. Nishio, T. Takano, Y. Hanaoka, C. Torii, H. Sekiguchi, S. kawashima, Shinohara, Y. Irimajiri. H. Koshiishi, T. Kosugi, Y. Shiomi, M. Sawa:

Magnetic Fields of Chromosphere and Corona Observed with New Nobeyama Radioheliograph

11:30-11:45 V. M. Bogod, B. E. Rjabov and S. R. Khafizov:

The Discovery of Slim Magnetic Field Structure in the Active Region Using the Solar Radio Observation

11:45-12:00 G. B. Gelfreikh et al:

The Methods of Measurements of the Solar Magnetic Fields Using Radio Observations with the RATAN-600

02:00-05:00 PM Excursion to the Summer Palace

07:00-09:30 PM

Colloquium Session 6: FLARES AND TRANSIENTS

Chairman, A. Bhatnagar

Invited Papers:

07:00-07:30 H. Wang:

Flows, Evolution of Magnetic Fields and Flares

07:30-08:00 J. C. Henoux:

Topological Singularities of the Magnetic Field and Solar Flares

Oral Contributed Papers:

08:00-08:15 Takeo Kosugi:

Hard X-ray Observations of Solar Flares by YOHKOH: A Preliminary Review

08:15-08:30 K. Shibata, Y. Ishido, L. Acton, K. Strong, T. Hirayama, Y. Uchida, A. McAllister, R. Matsumoto, S. Tsuneta, T. Shimizu, H. Hara, T. Sakurai, K. Ichimoto, Y. Nishino and Y. Ogawara:

Observations of X-ray Jets Using Yohkoh Soft X-ray Telescope

08:30-08:45 Fang Cheng, Su-ying Yin and Ming-de Ding:

Diagnostics of the Velocity Field in the Lower Atmospnere of Solar Flare

08:45-09:00 Vladimir Hayrapetyan:

Primary Energy Release in a Solar Flare 
09:00-09:15 A. Bhatnagar, N. Srivastava:

Magnetic Field Configuration in $\mathrm{H} \alpha$ Flare loops and Flaring Arches

09:15-09:30 Qijun Fu et al:

Conclusive Evidences for Reality of Solar Radio Fluctuation

FRIDAY, SEPT. 11

08:45-12:00 AM

Colloquium Session 7: MAGNETIC SHEAR AND ELECTRIC CURRENTS

Chairman, M. Hagyard

Invited Papers:

08:45-09:15 T. Forbes:

Model Prediction for Magnetic Shear Change During Solar Flares

09:15-09:30 Jingxiu Wang:

Analyses of Vector Magnetograms

09:30-10:45 Tea Break and Poster Session

Oral Contributed Papers:

10:45-11:00 V. I. Abramenko:

Systems of Vertical Electric Currents in Active Region

11:00-11:15 N. Seehafer:

Magnetic Field Line Topology in Solar Active Regions

11:15-11:30 Linxiang $W_{u}$ :

A Sufficient for Krall's Criterion and Its Modification

11:30-11:45 Xing Li and Y. Q. Hu :

Simulation of the Interaction of Two High Shear Magnetic Bipoles

11:45-12:00 Yuanzhang Lin, Xiaolei Wei and Hongqi Zhang:

Variations of Magnetic Fields and Electric Currents Associated with a

Solar Flare

02:00-05:00 PM

Colloquium Session 8: STRUCTURE AND ROLE OF EMERGING FLUX REGIONS

Chairman, C. Zwaan

Invited Papers:

02:00-02:30 D. Chou:

Structure of Emerging Flux Region

02:30-03:00 Y. Wang and N. R. Sheely. Jr.:

Flux Emergence and the Evolution of Large-scale Photospheric Field

Patterns

03:00-03:30 V. Gaizauskas:

Clustering of Active Region in Solar Activity

03:30-03:45 Tea Break

Oral Contributed Papers:

03:45-04:00 Karen L. Harvey:

The Global Properties of Active Regions

04:00-04:15 Robert F. Howard:

The Development of Sunspot Groups 
04:15-04:30 Pavel Ambroz:

Evolutionary Characteristics of Large-scale Magnetic and Velocity

Fields

04:30-04:45 H. Kurokawa and G. Kawai:

Morphological and Evolutional Characteristics of Surges Spouting from

Emerging Flux Regions

04:45-05:00 V. M. Grigoryev:

Background Magnetic Fields and Formation of Active Regions

07:00-09:30 PM Banquet

SATURDAY, SEPT. 12

08:45-12:00 AM

Colloquium Session 9: CONVECTIONS AND OSCILLATIONS IN ACTIVE REGIONS

Chairman, M. Makita

Invited Papers:

08:45-09:15 J. Thomas:

Sunspot Seismology: The Interaction of Solar p-mode with Sunspot

09:15-09:45 J. Harvey:

Active Region Oscillations

09:45-10:15 Tea Break

Oral Contributed Papers:

10:15-10:30 B. Fleck, F.-L. Deubner and W. Schmidt:

Observations of Waves and Oscillations in the Solar Magnetic Fine Structure

10:30-10:45 B. W. Lites, R. J. Rutten, W. Kalkofen:

Oscillations of the Magnetic Network

10:45-11:00 M. Ryutova, E. Priest:

The Interaction of Unsteady Acoustic Wave-packets with the Random Ensembles of Magnetic Flux Tubes

11:00-11:15 S. K. Solanki, I. Ruedi, D. Rabin:

Siphon Flow Across the Magnetic Neutral-line of an Active Region

11:15-11:30 A. V. Stepanov and Yu. T. Tsap:

Oscillations in Energy Release Volume: An Equivalent LRC-circuit

11:30-11:45 L. Nocera:

Fractal MHD Waves in the Solar Atmosphere

02:00-05:00 PM

Oral Contributed Papers:

02:00-02:15 G. Lustig and H. Wohl:

Plasma Motions Around Sunspots

02:15-02:30 P. Mein, N. Mein:

Flows in Active Regions: Imaging Spectroscopy in Chromosphere and Photosphere

Colloquium Session 10: WINDUP, FINAL REMARKS

Chairman, Guoxiang Ai

02:30 H. Zirin: Summary 